\title{
Virtual FTIR spectrometer and 3D visual display for a course in molecular spectroscopy
}

Lionel Bertrand, Sylvain Lefebvre, Serge Bernier, Dominic Villiard, Marie-Josée Leblanc, et al.

Lionel Bertrand, Sylvain Lefebvre, Serge Bernier, Dominic Villiard, MarieJosée Leblanc, Stéphane Proulx, "Virtual FTIR spectrometer and 3D visual display for a course in molecular spectroscopy," Proc. SPIE 9664, Ninth International Topical Meeting on Education and Training in Optics and Photonics, 96641J (24 October 2005); doi: 10.1117/12.2207541 
Ref ETOP007

\title{
Virtual FTIR spectrometer and 3D visual display for a course in molecular spectroscopy
}

Lionel Bertrand, Sylvain Lefebvre, Serge Bernier, Dominic Villiard, Marie-Josée Leblanc and Stéphane Proulx ${ }^{1}$

École Polytechnique de Montréal, Montréal, Canada

${ }^{1}$ Media42, Montréal.

\begin{abstract}
The use of a visual 3D display and a virtual spectrometer for the teaching of a course in molecular spectroscopy presents new possibilities. All the movements of the molecules are shown along with their relations with their characteristic spectra. The virtual laboratory simulates a true commercial spectrometer as well as a software of recognition. All the students simultaneously follow the laboratory under conditions very close to the reality of a modern industrial laboratory.
\end{abstract}

\section{Keywords}

Virtual laboratories ; simulation of a spectrometer ; molecular mode vibrations; infrared spectra library ; spectrum recognition ; student tracking.

\section{Summary}

Teaching a course in molecular spectroscopy is not an easy task. First, we must use concepts of molecular symmetry to relate the observed spectra to the quantum properties of the molecules, such as the creation or the modification of an electric dipole moment or the quantified energy levels. The visualization of these 3D symmetry properties is not easy, and the use of sticks and ball models has become complicated and obsolete today.

Second FTIR spectroscopy now involves very expensive equipment and signal analysers. Teaching laboratories often lack the number of spectrometers required for teaching large groups of students, and, even less, the expensive software to analyze and recognize the spectra. The result is that the students are not trained with modern apparatus and under the conditions normally met in their future work place. Sometimes they are also obliged to apply concepts in the laboratory, which they will learn only later in class.

The development of the information and communications technology (ICT) enables us to conceive the teaching of such courses differently. The first problem is easily solved by developing a 3D visual display with the six degrees of freedom which describe the translation and the rotation of the whole molecule. Moreover, the $3 \mathrm{~N}$ minus six (or five for linear molecule) degrees of freedom of a $\mathrm{N}$ atoms molecule can also be illustrated, showing all the movement of the atoms. So we can simulate the normal modes of vibration of a molecule or of a group and correlate them with their spectra. We have done this with the Java 3D language, and using the spectra from the National Institute of Standards and Technology (NIST) site. Several molecules were simulated, so that all the symmetry operations are presented, as well as some normal modes of vibration of 
different molecules and radicals. For each movement, its characteristic spectrum is shown. Some examples will be presented during the presentation.

The second ICT application that we developed is a virtual FTIR spectrometer. As in all the virtual laboratories which we have developed, this spectrometer is as close as possible to a real commercial $B O M E M \circledast 100$ spectrometer which we have in our laboratories. The idea is that the virtual laboratory will enable the student to operate such an apparatus very well when he meets it, or it can be used as practice for a future real laboratory (for an examination for example). We begin by showing a 3D animation which makes it possible for the student to have an idea of the real dimensions of the spectrometer and to stop at a detail which he wishes to observe better. He starts the apparatus and proceeds as if he were in front of the real spectrometer. All the operations necessary to obtain a spectrum with the BOMEM® 100 are simulated, including the nitrogen purge, the sample holder handling as well as the change of the samples, the scan, and the Fourier transform of interferograms. We can also zoom the spectra, compare them visually, add or divide different spectra. Almost all the functions used by the real software are simulated and applied to a few spectra. This point is important since we are not obliged to remake the software completely. We merely simulate their results for some spectra. It is indeed only a question of showing to the students what they can do with the spectrometer. The Fourier transform, data sampling and processing usually used with a FTIR spectrometer are simulated. Moreover, we simulate a pattern recognition software which makes it possible for the student to rapidly determine to which family of molecule the measured spectrum (alcohols, acids, esters, amines etc., or even more complicated molecules like polymers) belongs. This last point represents an important improvement on the real laboratory, since we do not have a pattern recognition software as it is too expensive for a use in a teaching laboratory.

A library representative of the principal families of molecules ( a hundred spectra) was assembled using spectra obtained from various references. The student practises the acquisition of the spectra with some real spectra obtained with the BOMEM® 100 spectrometer. Finally he must identify an unknown molecule starting from its infra-red spectrum.

To be as close as possible to reality, experimental missteps are possible (wrong sample, distorts step, error of family of molecule). They are indexed in another site, only accessible to the professor. 